Mariana de Sousa Ribero de Carvalho'

Ana Maria Gomes Pereira ${ }^{2}$

JoÃo AlFREDO MARTINS ${ }^{3}$

ReGinaldo Coelho Guedes LOPES ${ }^{1}$

\title{
Fatores preditores de recorrência do endometrioma ovariano após tratamento laparoscópico
}

\author{
Predictive factors for recurrence of ovarian endometrioma after \\ laparoscopic excision
}

Artigo Original

Palavras-chave

Endometriose

Ovário

Laparoscopia

Recidiva

Fatores de risco

Keywords

Endometriosis

Ovary

Laparoscopy

Recurrence

Risk factors

\section{Resumo}

OBJETIVO: Analisar os fatores que possam influenciar na recorrência do endometrioma ovariano após tratamento videolaparoscópico. MÉTODOS: Estudo de coorte retrospectivo. Foram avaliadas 129 pacientes submetidas à videolaparoscopia, entre 2003 e 2012 , para tratamento de endometrioma ovariano, com seguimento pós-operatório mínimo de 2 anos. Foi realizada ultrassonografia transvaginal para excluir persistência da lesão e identificação de recidiva. Para comparação de variáveis contínuas foi utilizado o teste t de Student, e para o teste de homogeneidade entre as proporções, o teste do $\chi^{2}$ ou exato de Fisher (para frequências menores do que cinco). Para a obtenção de fatores prognósticos ligados à recidiva foi aplicado o modelo de regressão linear multivariado. $\bigcirc$ nível de significância utilizado para a análise foi de 5\%. RESULTADOS: A taxa de recorrência foi de 18.6\%. $\bigcirc$ tamanho do endometrioma, a modalidade cirúrgica empregada e os dados demográficos como idade, índice de massa corpórea, presença de sintomas, tabagismo e prática de exercícios físicos não se associaram ao aumento da recorrência. Foi observado que a interrupção do tratamento clínico pós-operatório apresentou risco significativo para aumento da recorrência (OR 23,7; IC95\% 5,26-107,05; p=0,001). CONCLUSÃO: $\bigcirc$ uso contínuo de contracepção oral parece reduzir dramaticamente a taxa de recidiva de endometrioma ovariano. Neste estudo, a interrupção do tratamento foi o fator associado com maior taxa de recidiva da lesão após o tratamento videolaparoscópico.

\section{Abstract}

PURPOSE: To analyze the factors that might influence the recurrence of ovarian endometriomas after laparoscopic excision. METHODS: A retrospective cohort study. We evaluated 129 patients who underwent laparoscopic excision of ovarian endometriomas from 2003 to 2012 and who were followed up for at least two years after surgery. Vaginal ultrasound was repeated to exclude persistent lesion and to identify recurrence. The Student's t-test was used to compare continuous variables and the $\chi^{2}$ or Fischer exact test (for values of less than five) was used to test homogeneity between proportions. A logistic regression model for multivariate proportional hazards was used to analyze predictors of long-term outcome. The level of significance was set at $5 \%$ in all analyses. RESULTS: The overall rate of ovarian endometrioma recurrence was 18.6\%. Endometrioma diameter, surgical procedure techniques and demographic data such as age, presenting symptoms, body mass index, smoking and physical exercise habits were not associated with recurrence, whereas interruption of postoperative medical treatment was significantly correlated with a higher recurrence rate $\mid O R 23.7 ; 95 \% \mathrm{Cl} 5.26-107.05$; $p=0.0011$. CONCLUSION: Current oral contraceptive use appears to be associated with a dramatic reduction in the risk of recurrence of ovarian endometriotic cysts. Treatment interruption was associated with a higher recurrence rate of ovarian endometrioma after laparoscopic treatment.
Correspondência

Mariana de Sousa Ribeiro de Carvalho Serviço de Ginecologia e Obstetríia, Hospital do Servidor Público Estadual "Francisco Morato de Oliveira" Rua Pedro de Toledo, 1800, $4^{\circ}$ andar, ala par

Vila Clementino (EP: 04039-901

São Paulo (SP), Brasi

Recebido

28/10/2014

Aceito com modificaçōes

05/01/2015
Departamento de Ginecologia e Obstetrícia do Hospital do Servidor Público Estadual de São Paulo "Francisco Morato de Oliveira" HSPE-FMO - São Paulo (SP), Brasil.

'Serviço de Ginecologia e Obstetrícia, Hospital do Servidor Público Estadual de São Paulo "Francisco Morato de Oliveira" - HSPE-FMO - São Paulo (SP), Brasil.

2Setor de Endometriose e Dor Pélvica Crônica, Serviço de Ginecologia e Obstetrícia, Hospital do Servidor Público Estadual de São Paulo "Francisco Morato de Oliveira" - HSPE-FMO - São Paulo (SP), Brasil.

${ }^{3}$ Seção de Ginecologia, Serviço de Ginecologia e Obstetrícia, Hospital do Servidor Público Estadual de São Paulo "Francisco Morato de Oliveira" - HSPE-FMO - São Paulo (SP), Brasil.

Conflito de interesses: não há. 


\section{Introdução}

A endometriose afeta, aproximadamente, 5 a $12 \%$ das mulheres na menacme, com prevalência ainda mais significativa, em torno de $50 \%$, nas pacientes com infertilidade ${ }^{1,2}$. O endometrioma ovariano é a lesão mais frequente, incidindo, em média, em $55 \%$ das pacientes portadoras dessa afecção ${ }^{3}$.

A videolaparoscopia com exérese da cápsula do cisto endometriótico é o tratamento mais frequentemente realizado, com evidências na literatura de que possa resultar em menor taxa de recorrência da lesão $0^{4-6}$, quando comparada à fenestração ou drenagem do cisto $^{7-9}$. Uma metanálise de sete ensaios clínicos randomizados demonstra que a exérese da cápsula foi associada ao menor risco de recorrência dos sintomas, com odds ratio (OR) de 0,2 e menor risco de recidiva do endometrioma $(\mathrm{OR}=0,5)$ após a cirurgia quando comparada à fenestração/coagulação ${ }^{10}$. Entretanto, como esse resultado não é compartilhado por todos os estudos $^{11}$, a melhor técnica cirúrgica para abordagem dos cistos endometrióticos ainda precisa ser discutida.

Vários autores recomendam o tratamento com supressão hormonal no pós-operatório de cirurgia conservadora para endometrioma ovariano, buscando-se amenorreia medicamentosa, para diminuir a taxa de recorrência e os sintomas secundários à endometriose. Dentre os métodos hormonais empregados, os contraceptivos orais são excelente opção por seu baixo custo, segurança e tolerabilidade ${ }^{4,6,12,13}$. Um ensaio clínico prospectivo randomizado visando observar a correlação entre uso de contracepção e recorrência dos endometriomas após a videolaparoscopia distribuiu as 217 pacientes em três grupos: não usuárias, usuárias de contracepção cíclica e usuárias de forma contínua. O estudo demonstra taxa de recorrência de $29 \%$ no grupo das não usuárias, $14,7 \%$ nas usuárias cíclicas $(\mathrm{p}=0,02)$ e $8,2 \%$ nas que usaram contracepção monofásica contínua $(\mathrm{p}=0,003)^{3}$.

A recorrência após tratamento cirúrgico permanece elevada e parece relacionada com a duração do seguimento $^{14}$, com taxas que variam de 6 a $30 \%{ }^{7,13}$ em $1-5$ anos $^{15}$, diferenças justificadas pelo tempo de seguimento das pacientes, modalidade cirúrgica empregada e critérios adotados para definição de recorrência em cada estudo ${ }^{8,15,16}$. Desta forma, muito ainda se discute sobre as causas de recidiva e fatores predisponentes, os quais ainda permanecem incertos.

De forma geral, quanto mais avançada a endometriose, maior a chance de recidiva da doença ${ }^{15}$. Tamanho dos $\operatorname{cistos}^{14,17}$, escore revisado da Sociedade Americana de Medicina Reprodutiva (rASRM) ${ }^{2,14,18}$ elevado, idade na época da cirurgia ${ }^{19}$, presença de sintomas ${ }^{20}$ e cirurgia laparoscópica com drenagem isolada do cisto $^{7}$ foram reportados como fatores de risco para recidiva. Fatores correlacionados com a severidade da endometriose, como tratamento clínico pré-operatório ${ }^{17}$, cirurgia prévia para endometriose $^{2,14}$ e tempo de seguimento das pacientes ${ }^{21}$ foram também identificados como fatores de risco em outras publicações, ao passo que a ocorrência gestação $0^{2,17,19}$ e o uso de contraceptivos orais ${ }^{12}$ são considerados fatores protetores para recidiva, reforçando a afirmativa de que os fatores preditores e os protetores de recorrência de endometrioma ovariano reportados na literatura ainda são conflitantes.

O objetivo deste trabalho é analisar os fatores preditores de recorrência do endometrioma ovariano após tratamento cirúrgico videolaparoscópico.

\section{Métodos}

Trata-se de estudo de coorte retrospectivo, envolvendo 195 casos de endometrioma ovariano, tratados por videolaparoscopia e acompanhados entre janeiro de 2003 e junho de 2012, com seguimento mínimo por 2 anos, no Ambulatório de Endometriose e Dor Pélvica Crônica do Hospital do Servidor Público Estadual de São Paulo "Francisco Morato de Oliveira" (HSPE-FMO). O estudo foi aprovado pelo Comitê de Ética em Pesquisa da mesma instituição.

Foram incluídas pacientes na menacme, com diagnóstico de endometrioma ovariano, que foram submetidas à drenagem do cisto ou ressecção da cápsula por videolaparoscopia e que tiveram seguimento pós-cirúrgico por, no mínimo, dois anos. Foram excluídas as pacientes na pós-menopausa, as que perderam ou tiveram um seguimento menor do que dois anos; aquelas submetidas à ooforectomia e pacientes com lesões persistentes. Do total, 129 pacientes preencheram os critérios de inclusão.

A cirurgia laparoscópica foi realizada empregando-se a técnica tradicional, com inspeção de toda cavidade abdominal e, onde houve necessidade, foi realizada lise de aderências perianexiais. Para exérese da cápsula do endometrioma foi feita incisão cortical ovariana e definiu-se o plano de clivagem entre o ovário e o cisto. Posteriormente, o cisto foi enucleado com sua cápsula e liberado do ovário com o auxílio de pinças de preensão e movimento de tração e contra-tração e removido da cavidade através do trocater ou com o auxílio de endobags, com subsequente hemostasia dos focos sangrantes. Somente nos casos de distorção da arquitetura ovariana por cistos volumosos, infiltração da cápsula do cisto com perda do plano de clivagem e grandes aderências perianexiais que dificultavam mobilizar o ovário e impossibilitavam o acesso ao endometrioma, optava-se pela drenagem simples do cisto. Nesses casos, após a incisão cortical, os mesmos foram drenados e seu conteúdo aspirado. Rotineiramente, cauterizava-se 
o leito remanescente do endometrioma. Caso houvesse sangramento no sítio da incisão, realizava-se hemostasia com corrente elétrica bipolar

A ultrassonografia transvaginal foi solicitada no terceiro mês após a cirurgia para excluir eventual persistência da lesão e repetida no sexto mês e anualmente para identificar recidivas. O seguimento foi anual para pacientes assintomáticas com parâmetros ultrassonográficos sem alterações e semestral caso houvesse evidência de cisto simples.

Durante o seguimento, foi considerada recorrência da doença ovariana caso houvesse surgimento de novo cisto persistente com características ultrassonográficas sugestivas de endometrioma, ou se fosse diagnosticado endometrioma com nova abordagem cirúrgica com confirmação anatomopatológica.

Instituiu-se tratamento hormonal para todas as pacientes (progestagênio isolado ou métodos combinados de estrogênio e progestagênio) objetivando indução de amenorreia medicamentosa, após a cirurgia.

As variáveis epidemiológicas analisadas foram: idade, índice de massa corpórea (IMC), presença de sintomas (dispareunia, dor acíclica e dismenorreia), tabagismo e prática de atividade física. Além disso, foram estudados o tamanho do endometrioma, a cirurgia realizada (drenagem do cisto ou exérese da cápsula), bilateralidade e intervalo sem tratamento clínico pós-operatório.

Inicialmente todas as variáveis foram analisadas descritivamente. As variáveis quantitativas foram apresentadas através de média, desvio padrão (DP), mediana e valores mínimos e máximos. Para as variáveis qualitativas foram calculadas frequências absolutas e relativas.

Os dados foram analisados com uso do software Statistical Package for the Social Sciences (SPSS) versão 17.0 para Windows. Para a comparação de médias dos dois grupos foi empregado o teste $t$ de Student e para o teste de homogeneidade entre as proporções foi utilizado o teste do $\chi^{2}$ ou exato de Fisher (para frequências menores do que cinco). Para obtenção de fatores prognósticos associados à recidiva foi aplicado o modelo de regressão linear multivariado para valores de $\mathrm{p}<0,2$ obtidos na análise univariada. O nível de significância utilizado para os testes foi de $5 \%$.

\section{Resultados}

A amostra estudada foi composta por mulheres com média de idade de 36,8 $\pm 5,5$ anos e índice de massa corpórea de $25,2 \pm 5,6 \mathrm{~kg}$. Sintomas relacionados à endometriose (dispareunia, dor acíclica e dismenorreia) estavam presentes em $95,3 \%$ das pacientes, o tabagismo em $7,3 \%$, a bilateralidade dos cistos em $37,9 \%$ e a prática de exercícios físicos em $35,2 \%$.

O tamanho médio dos cistos foi de 4,7 $42,4 \mathrm{~cm}$. Em relação à técnica cirúrgica empregada, 57,3\% das pacientes se submeteram à exérese da cápsula e 42,6\% à drenagem do cisto.

O grupo de pacientes com recidiva não apresentou diferença significativa em relação ao tamanho do endometrioma $(\mathrm{p}=0,8)$ e aos dados demográficos estudados, como idade, índice de massa corpórea, presença de sintomas e hábitos como tabagismo e prática de exercícios físicos (Tabela 1).

O tempo médio de seguimento foi de $66,9 \pm 34,7$ meses.

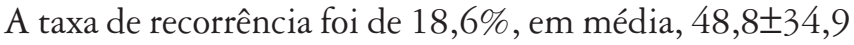
meses após a cirurgia.

Com relação aos fatores prognósticos, foi observado que a bilateralidade $(\mathrm{p}=0,3)$, cisto com diâmetro maior ou igual a $4 \mathrm{~cm}(\mathrm{p}=0,3)$ e o tipo de cirurgia realizada $(\mathrm{p}=0,08)$ não demonstraram associação com recidiva.

Do total, 54 pacientes $(41,9 \%)$ interromperam o tratamento hormonal pós-operatório de modo permanente ou transitório. Houve interrupção do tratamento por 22 pacientes $(91,5 \%)$ no grupo de recorrência, com tempo médio de descontinuidade por $26,6 \pm 18,4$ meses. O grupo de pacientes com recidiva do endometrioma ovariano apresentou diferença significativa em relação ao intervalo sem tratamento clínico (OR 23,7; IC95\% 5,3-107; $\mathrm{p}=0,001)$, como exposto na Tabela 2.

Tabela 1. Análise dos dados demográficos segundo o grupo de pacientes com recidiva do cisto endometriótico

\begin{tabular}{lcc}
\hline \multirow{2}{*}{ Variável } & \multicolumn{2}{c}{ Recidiva } \\
\cline { 2 - 3 } & Não & Sim \\
\hline Idade (anos) (média \pm DP) & $36,8 \pm 5,7$ & $37,2 \pm 5,2$ \\
Índice de massa corpórea (média \pm DP) & $25,2 \pm 6,0$ & $25,2 \pm 3,9$ \\
Sintomas n (\%) & & \\
$\quad$ Não & $6(5,7)$ & - \\
$\quad$ Sim & $99(94,3)$ & $24(100)$ \\
Tabagísmo n (\%) & & \\
$\quad$ Não & $92(92,0)$ & $21(95,5)$ \\
$\quad$ Simm & $8(8,0)$ & $1(4,6)$ \\
Exercícios físicos n (\%) & & $14(66,7)$ \\
$\quad$ Não & $63(64,3)$ & $7(33,3)$ \\
$\quad$ Sim & $35(35,7)$ & \\
\hline
\end{tabular}

DP: desvio padrão.

Tabela 2. Avaliação dos fatores prognósticos segundo o grupo de pacientes com recidiva do endometrioma ovariano

\begin{tabular}{|c|c|c|c|c|}
\hline \multirow[b]{2}{*}{ Variável } & \multicolumn{2}{|c|}{ Recidiva } & \multirow[b]{2}{*}{ OR } & \multirow[b]{2}{*}{ IC95\% } \\
\hline & $\begin{array}{c}\text { Não } \\
\text { n (\%) }\end{array}$ & $\begin{array}{c}\operatorname{Sim}_{\mathrm{n}}(\%) \\
\text { (\%) }\end{array}$ & & \\
\hline Bilateralidade & $42(40)$ & $7(29,2)$ & 0,6 & $0,2-1,6$ \\
\hline Cisto $\geq 4 \mathrm{~cm}$ & $51(51)$ & $9(39,1)$ & 0,6 & $0,2-1,5$ \\
\hline Drenagem & $41(39,1)$ & $14(58,3)$ & 2,2 & $0,9-5,4$ \\
\hline Ressecção do cisto & $64(61)$ & $10(42,0)$ & 0,5 & $0,2-1,1$ \\
\hline Interrupção do trafamento & $32(32)$ & $22(92,0)$ & 23,7 & $5,3-107$ \\
\hline
\end{tabular}

OR: odds ratio; IC95\%: intervalo de confiança de $95 \%$. 
Aplicando-se a regressão linear multivariada para as variáveis com $\mathrm{p}<0,2$ na análise univariada, foi demonstrado que, independente da idade da paciente $(\mathrm{p}=0,5) \mathrm{e}$ da técnica cirúrgica empregada $(\mathrm{p}=0,08)$, a única variável associada com a recorrência foi a interrupção do tratamento clínico pós-operatório $(\mathrm{p}<0,001)$.

\section{Discussão}

A recorrência dos endometriomas ovarianos após tratamento videolaparoscópico ainda permanece elevada ${ }^{22}$ e sua prevenção incerta porque a patogênese da recidiva ainda é desconhecida ${ }^{23}$. Vários autores defendem que os tratamentos clínico e cirúrgico adequados minimizam o dano ovariano, amenizam os sintomas da doença e, possivelmente, reduzem a recidiva da lesão $0^{12,24,25}$.

Em revisão sistemática da literatura, levando-se em conta o emprego da cirurgia laparoscópica para o tratamento do endometrioma ovariano, a exérese da cápsula tem se mostrado superior à drenagem isolada do cisto em relação à recidiva ${ }^{7,8,11}$ e ao melhor controle dos sintomas ${ }^{6,24}$.

Não há consenso de que a bilateralidade e o tamanho dos endometriomas prediz maior taxa de recorrência. Um estudo retrospectivo com 224 pacientes demonstrou que o envolvimento uni ou bilateral ovariano não influencia no aumento dessas taxas, ao passo que o tamanho do maior cisto visto no intraoperatório influencia os índices de recorrência ${ }^{17}$.

Uma análise retrospectiva de 62 mulheres em idade reprodutiva que apresentavam endometrioma recorrente demonstrou que $80,6 \%$ recidivaram no ovário tratado cirurgicamente, $11,3 \%$ no ovário contralateral não tratado e $8,1 \%$ em ambos os ovários 8 . Cogita-se que a recorrência dos cistos deve estar relacionada a focos residuais no local já tratado ${ }^{26,27}$ ou à reimplantação e ao crescimento do endométrio ectópico ${ }^{15,16}$. No presente estudo, a técnica cirúrgica empregada, a bilateralidade e o diâmetro dos cistos não influenciaram as taxas de recorrência do endometrioma ovariano.

Nesse contexto, um ponto em comum entre as publicações recentes é o uso de contracepção oral após o tratamento cirúrgico conservador dos cistos endometrióticos ${ }^{3,12,21,27,28}$. Seu emprego se justifica pela indução da apoptose, redução da proliferação endometrial e inibição da ovulação, o que diminui a dor relatada ${ }^{23,27}$ e o desenvolvimento dos endometriomas ovarianos ${ }^{5}$. O efeito oposto é observado nas pacientes que optam pela descontinuidade, mesmo que transitória, do método hormonal supressor da ovulação utilizado ${ }^{5,16}$.

Um estudo de coorte retrospectivo envolvendo 87 pacientes com endometrioma reportou menor taxa de recidiva entre as que usaram contracepção oral contínua no pós-operatório, com taxas ainda menores naquelas que mantiveram seu uso por mais de 24 meses, quando comparadas àquelas que interromperam seu uso no fim do seguimento ou àquelas que nunca usaram. As taxas de recidiva relatadas foram 2,9; 14,3 e 43,5\%, respectivamente, nos grupos de uso de contracepção oral sem interrupção, uso com interrupção e grupo das que nunca usaram ${ }^{18}$.

Achado similar foi publicado em estudo coorte no qual se observou que $94 \%$ das pacientes em uso de anticoncepção oral contínua, com seguimento por 36 meses, não tiveram recorrência, comparadas com uma taxa de $51 \%$ naquelas que não receberam esse tratamento 9 . Em contrapartida, um estudo randomizado controlado envolvendo 259 pacientes submetidas à exérese da cápsula do endometrioma não publica resultado semelhante. As pacientes foram randomizadas para receberem placebo $(n=65)$, agonista do hormônio liberador de gonadotrofina $(\mathrm{GnRH})(\mathrm{n}=65)$, contracepção contínua de baixa dose $(n=64)$ e dieta $(\mathrm{n}=65)$ por 6 meses com seguimento por 18 meses. Foi observado que não houve diferença nas taxas de recorrência quando comparados a utilização de método hormonal pós-operatório, placebo e dieta ${ }^{16}$.

No presente estudo, como todas as pacientes receberam tratamento hormonal em algum momento do seguimento, não se pode observar a taxa de recidiva naquelas sem qualquer tratamento, o que pode subestimar a taxa de recorrência apresentada. Houve aumento de recidiva naquelas pacientes que interromperam o tratamento clínico pós-cirúrgico, com chance de 23,7 vezes maior de recorrência do endometrioma nesse grupo.

Outra limitação de um estudo retrospectivo recai nos vieses de obtenção dos dados coletados. Por falta de informações precisas não se pode observar a taxa de recidiva nas pacientes que utilizaram algum método hormonal antes da cirurgia. Vale ainda lembrar que as cirurgias não foram realizadas pelo mesmo médico, e a experiência e destreza individual podem interferir nos resultados cirúrgicos apresentados. Em contrapartida, destaca-se a análise de dez variáveis possivelmente relacionadas à recorrência, com um longo período de seguimento das pacientes do estudo.

É mister esclarecer as pacientes sobre as elevadas taxas de recidiva após tratamento videolaparoscópico e a importância de um seguimento pós-cirúrgico rigoroso. O uso contínuo de contracepção oral após a cirurgia parece reduzir dramaticamente a taxa de recidiva de endometrioma ovariano.

Predizer, portanto, algum fator que seja relacionado à recorrência da doença permite triar as pacientes de maior risco, empregar a melhor forma de tratamento e segui-las com maior cautela. 
1. Nácul AP, Spritzer PM. [Current aspects on diagnosis and treatment of endometriosis]. Rev Bras Ginecol Obstet. 2010;32(6):298-307. Portuguese.

2. Porpora MG, Pallante D, Ferro A, Crisafi B, Bellati F, Benedetti PP. Pain and ovarian endometrioma recurrence after laparoscopic treatment of endometriosis: a long-term prospective study. Fertil Steril. 2010;93(3):716-21.

3. Seracchioli R, Mabrouk M, Frascà C, Manuzzi L, Montanari G, Keramyda $A$, et al. Long-term cyclic and continuous oral contraceptive therapy and endometrioma recurrence: a randomized controlled trial. Fertil Steril. 2010;93(1):52-6.

4. Bourdel N, Roman H, Mage G, Canis M. [Surgery for the management of ovarian endometriomas: from the physiopathology to the pre-, peri- and postoperative treatment]. Gynecol Obstet Fertil. $2011 ; 39(12): 709-21$. French.

5. Ouchi N, Akira S, Mine K, Ichikawa M, Takeshita T. Recurrence of ovarian endometrioma after laparoscopic excision: risk factors and prevention. J Obstet Gynaecol Res. 2014;40(1):230-6.

6. Sengoku K, Miyamoto T, Horikawa M, Katayama H, Nishiwaki $\mathrm{K}$, Kato $\mathrm{Y}$, et al. Clinicopathologic risk factors for recurrence of ovarian endometrioma following laparoscopic cystectomy. Acta Obstet Gynecol Scand. 2013;92(3):278-84.

7. Hart RJ, Hickey M, Maouris P, Buckett W. Excisional surgery versus ablative surgery for ovarian endometriomata. Cochrane Database Syst Rev. 2008;(2):CD004992.

8. Exacoustos C, Zupi E, Amadio A, Amoroso C, Szabolcs B, Romanini $M E$, et al. Recurrence of endometriomas after laparoscopic removal: sonographic and clinical follow-up and indication for second surgery. J Minim Invasive Gynecol. 2006;13(4):281-8.

9. Vercellini $P$, de Matteis S, Somigliana E, Buggio L, Frattaruolo $M P$, Fedele L. Long-term adjuvant therapy for the prevention of postoperative endometrioma recurrence: a systematic review and meta-analysis. Acta Obstet Gynecol Scand. 2013;92(1):8-16.

10. Dan H, Limin F. Laparoscopic ovarian cystectomy versus fenestration/ coagulation or laser vaporization for the treatment of endometriomas: a meta-analysis of randomized controlled trials. Gynecol Obstet Invest. 2013;76(2):75-82.

11. Moscarini M, Milazzo GN, Assorgi C, Pacchiarotti A, Caserta D. Ovarian stripping versus cystectomy: recurrence of endometriosis and pregnancy rate. Arch Gynecol Obstet. 2014;290(1):163-7.

12. Vercellini $P$, Somigliana $E$, Daguati R, Vigano $P$, Meroni $F$, Crosignani PG. Postoperative oral contraceptive exposure and risk of endometrioma recurrence. Am J Obstet Gynecol. 2008; 198(5):504.e1-5.

13. Diwadkar $G B$, Falcone $T$. Surgical management of pain and infertility secondary to endometriosis. Semin Reprod Med. 2011 ;29(2): 124-9.

14. Busacca M, Marana R, Caruana P, Candiani M, Muzii L, Calia $C$, et al. Recurrence of ovarian endometrioma after laparoscopic excision. Am J Obstet Gynecol. 1999; 180(3 Pt 1):519-23.
15. Koga K, Osuga Y, Takemura Y, Takamura M, Taketani Y. Recurrence of endometrioma after laparoscopic excision and its prevention by medical management. Front Biosci. 2013;5:676-83.

16. Sesti F, Capozzolo T, Pietropolli A, Marziali M, Bollea MR, Piccione E. Recurrence rate of endometrioma after laparoscopic cystectomy: a comparative randomized trial between post-operative hormonal suppression treatment or dietary therapy vs. placebo. Eur J Obstet Gynecol Reprod Biol. 2009; 147(1):72-7.

17. Koga K, Takemura Y, Osuga Y, Yoshino O, Hirota Y, Hirata T, et al. Recurrence of ovarian endometrioma after laparoscopic excision. Hum Reprod. 2006;21(8):2171-4.

18. Takamura M, Koga K, Osuga $Y$, Takemura $Y$, Hamasaki $K$, Hirota $Y$, et al. Post-operative oral contraceptive use reduces the risk of ovarian endometrioma recurrence after laparoscopic excision. Hum Reprod. 2009;24(12):3042-8.

19. Sengoku K, Miyamoto T, Horikawa M, Katayama H, Nishiwaki $K$, Kato $Y$, et al. Clinicopathologic risk factors for recurrence of ovarian endometrioma following laparoscopic cystectomy. Acta Obstet Gynecol Scand. 2013;92(3):278-84.

20. Maul LV, Morrision JE, Schollmeyer T, Alkatout I, Mettler L. Surgical therapy of ovarian endometrioma: recurrence and pregnancy rates. JSLS. 2014;18(3):e2014.00223.

21. Vercellini P, Fedele L, Aimi G, de Giorgi O, Consonni D, Crosignani PG. Reproductive performance, pain recurrence and disease relapse after conservative surgical treatment for endometriosis: the predictive value of the current classification system. Hum Reprod. 2006;21(10):2679-85.

22. Kim ML, Kim JM, Seong SJ, Lee SY, Han M, Cho YJ. Recurrence of ovarian endometrioma after second-line, conservative, laparoscopic cyst enucleation. Am J Obstet Gynecol. 2014;210(3):216.e1-6.

23. Gelbaya TA, Nardo LG. Evidence-based management of endometrioma. Reprod Biomed Online. 2011 ;23(1):15-24.

24. Rimbach S, Ulrich U, Schweppe KW. Surgical therapy of endometriosis: challenges and controversies. Geburtshilfe Frauenheilkd. 2013;73(9):918-23.

25. Shah DK, Mejia RB, Lebovic DI. Effect of surgery for endometrioma on ovarian function. J Minim Invasive Gynecol. 2014;21 (2):203-9.

26. Somigliana E, Benaglia L, Vercellini P, Paffoni A, Ragni G, Fedele L. Recurrent endometrioma and ovarian reserve: biological connection or surgical paradox? Am J Obstet Gynecol. $2011 ; 204(6): 529$. e 1-5.

27. Guo SW. Recurrence of endometriosis and its control. Hum Reprod Update. 2009;15(4):441-61.

28. Vercellini $P$, Somigliana $E$, Viganò $P$, de Matteis $S$, Barbara $G$, Fedele L. Post- operative endometriosis recurrence: a plea for prevention based on pathogenetic, epidemiological and clinical evidence. Reprod Biomed Online. 2010;21 (2):259-65. 